\title{
Quasiperiodic spin chains in a magnetic field
}

\author{
M. Arlego, ${ }^{1}$ D. C. Cabra,${ }^{1,2}$ and M. D. Grynberg ${ }^{1}$ \\ ${ }^{1}$ Departamento de Física, Universidad Nacional de La Plata, C.C. 67, (1900) La Plata, Argentina \\ ${ }^{2}$ Facultad de Ingeniería, Universidad Nacional de Lomas de Zamora, Cno. de Cintura y Juan XXIII, (1832) \\ Lomas de Zamora, Argentina
}

(Received 10 April 2001; published 12 September 2001)

\begin{abstract}
We study the interplay between a (quasi) periodic coupling array and an external magnetic field in a spin- $\frac{1}{2}$ $X X Z$ chain. A new class of magnetization plateau is obtained by means of Abelian bosonization methods which gives rise to a sufficient quantization condition. The investigation of magnetic phase diagrams via exact diagonalization of finite clusters finds a complete agreement with the continuum treatment in a variety of situations.
\end{abstract}

DOI: 10.1103/PhysRevB.64.134419

PACS number(s): 75.60.Ej, 71.10.Fd, 71.10.Pm

The magnetic properties of quasicrystals have become a fundamental issue of study since their discovery in $1984 .{ }^{1} \mathrm{~A}$ variety of theoretical efforts, ranging from renormalization group $(\mathrm{RG})$ analysis of Ising models in Penrose lattices ${ }^{2}$ to exact solutions of both Ising and $X Y$ Fibonacci spin chains $^{3-5}$ have revealed fairly intricate magnetic orderings associated with the quasiperiodicity of these structures. The nonmetallic spin-exchange mechanism implicit in those studies has been evidenced in recently synthesized rare-earth (RE) ZnMg-RE quasicrystals (see, e.g., Ref. 6) whose RE elements have well localized $4 f$ magnetic moments.

Bolstered by these latter findings and as a further step within the line of the local moment descriptions referred to above, here we consider the ordering of quasiperiodic spin- $\frac{1}{2}$ $X X Z$ chains in a magnetic field to elucidate the quantization conditions of massive spin excitations or magnetization plateaus. In periodic systems, this issue has received systematic attention in the last few years from both experimental and theoretical points of view (see, e.g., Ref. 7). In this paper, we are specifically interested in studying the antiferromagnetic system

$$
\begin{aligned}
H_{q p}= & J \sum_{n}\left(1+\epsilon_{n}\right)\left(S_{n}^{x} S_{n+1}^{x}+S_{n}^{y} S_{n+1}^{y}+\Delta S_{n}^{z} S_{n+1}^{z}\right) \\
& -h \sum_{n} S_{n}^{z},
\end{aligned}
$$

where $S^{x}, S^{y}, S^{z}$ denote the spin- $\frac{1}{2}$ matrices involved in the standard $X X Z$ Hamiltonian $\left(\epsilon_{n}=0\right)$ in a magnetic field $h$ applied along the anisotropy direction $(|\Delta| \leqslant 1)$. Here, the coupling modulation is introduced via the $\epsilon_{n}$ parameters defined as $\epsilon_{n}=\Sigma_{\nu} \delta_{\nu} \cos \left(2 \pi \omega_{\nu} n\right)$, so quasiperiodicity arises upon choosing an irrational subset of frequencies $\omega_{\nu}$ with amplitudes $\delta_{\nu}$.

The interest of Eq. (1) stems partly from the widespread applications of $1 d$ Hamiltonians in the description of artificially grown quasiperiodic heterostructures, ${ }^{8}$ quantum dot crystals, ${ }^{9}$ and magnetic multilayers. ${ }^{10}$ Also, recent investigations of quasiperiodicity involving either the couplings ${ }^{11}$ or the magnetic field, ${ }^{12}$ have been addressed using Abelian bosonization along with RG and numerical techniques. Here we focus on the combined effect of a quasiperiodic exchange modulation under a uniform magnetic field.

Of particular importance are the rational frequencies of Eq. (1), not only as a way to approach the quasiperiodic limit, but also because they allow for a thorough numerical verification of a novel situation (see Refs. 13-15 for related work). As we shall see, although the allowed fractional plateaus predicted in the present case fall into the classification provided by the generalized Lieb-Schultz-Mattis theorem, ${ }^{16}$ a bosonization approach to Eq. (1) yields an alternative scenario not envisaged in previous studies, ${ }^{17,18}$ This will be reflected in the appearance of magnetization plateaus associated to each of the frequencies present in Eq. (1). To strengthen the potential interest of our results, we show how a simple two frequency model exhibits a magnetization curve with two wide plateaus at $1 / 4$ and $3 / 4$ of saturation, a situation which is highly reminiscent of that observed in magnetization experiments on $\mathrm{NH}_{4} \mathrm{CuCl}_{3} \cdot{ }^{19}$

Following the standard bosonization procedure (see, e.g., Ref. 20), the continuum limit of the $X X Z$ Hamiltonian in the presence of an external magnetic field $h$ is given by the Tomonaga-Luttinger Hamiltonian

$$
H=\frac{1}{2} \int d x\left(v K\left(\partial_{x} \widetilde{\phi}\right)^{2}+\frac{v}{K}\left(\partial_{x} \phi\right)^{2}\right) .
$$

The bosonic field $\phi$ and its dual $\widetilde{\phi}$ are given by the sum and difference of the light-cone components, respectively. The constant $K=K(\langle M\rangle, \Delta)$ governs the conformal dimensions of the bosonic vertex operators and can be obtained exactly from the Bethe-Ansatz solution of the $X X Z$ chain (see, e.g., Ref. 18 for a detailed summary). One has $K=1$ for the SU(2) symmetric case $(\Delta=1)$ and it is related to the radius $R$ of Ref. 18 by $K^{-1}=2 \pi R^{2}$. In terms of these fields, the spin operators read

$$
\begin{gathered}
S_{x}^{z}=\frac{1}{\sqrt{2 \pi}} \partial_{x} \phi+a: \cos \left(2 k_{F} x+\sqrt{2 \pi} \phi\right):+\frac{\langle M\rangle}{2}, \\
S_{x}^{ \pm}=(-1)^{x}: e^{ \pm i \sqrt{2 \pi} \tilde{\phi}}\left[b \cos \left(2 k_{F} x+\sqrt{2 \pi} \phi\right)+c\right]:,
\end{gathered}
$$

where the colons denote normal ordering with respect to the ground state with magnetization $\langle M\rangle$. The Fermi momentum 
$k_{F}$ is related to the magnetization of the chain as $k_{F}=(1$ $-\langle M\rangle) \pi / 2$. Either the $X X Z$ anisotropy or an external magnetic field modify the scaling dimensions of the physical fields through $K$ and the commensurability properties of the spin operators, as can be seen from Eqs. (3) and (4). The nonuniversal constants $a, b$, and $c$ can be in general computed numerically (see, e.g., Ref. 21, for the case of zero magnetic field) and in particular the constant $b$ has been obtained exactly in Ref. 22.

For the sake of clarity, let us first consider the effect introduced by a single frequency term in Eq. (1), i.e., $\epsilon_{n}$ $=\delta \cos (2 \pi \omega n)$. Thus using Eqs. (3) and (4) it follows that the relevant part of the interaction term in $H_{q p}=H+H_{i n t}$ reads

$$
\begin{aligned}
H_{\text {int }}= & \sum_{x} \cos (2 \pi \omega x)\left[\lambda_{1}\left(\partial_{x} \widetilde{\phi}\right)^{2}+\lambda_{2}\left(\partial_{x} \phi\right)^{2}+\lambda_{3} \cos \left(2 k_{F} x\right.\right. \\
& \left.+\sqrt{2 \pi} \phi)+\lambda_{4} \sin \left(2 k_{F} x+\sqrt{2 \pi} \phi\right)\right],
\end{aligned}
$$

where $\lambda_{i} \propto \delta, \quad i=1, \ldots, 4$.

As in previous analysis ${ }^{16-18}$ one can readily obtain the necessary quantization condition for the appearance of a plateau by looking at the commensurability of the relevant operators. In the present case we need only to consider the vertex operators $\exp \pm i \sqrt{2 \pi} \phi$ of scaling dimension $d$ $=1 /\left(4 \pi R^{2}\right)$. Therefore we obtain that $\langle M\rangle$ should satisfy

$$
\langle M\rangle= \pm(2 \omega-1),
$$

in order for a plateau to be present. The fulfillment of this condition opens a spin-gap excitation since the operator in question is relevant at least for $0<\Delta<1$. In the region -1 $<\Delta<0$ a critical curve appears which can be determined from the Bethe-Ansatz solution for $R(\langle M\rangle, \Delta){ }^{18}$ Furthermore, the gap width can be easily computed to scale as $\delta^{1 /(2-d)}$. Notice that the perturbations $\lambda_{1,2}$ do not play a role here since they are incommensurate whenever Eq. (6) holds.

In fact, these expectations turn out to apply well above the weak-coupling regime discussed so far. In Fig. 1 we show the magnetization phase diagram resulting from exact diagonalization of fairly large $X X$ chains $\left(10^{5}\right.$ spins $)$ by setting $\omega=13 / 21$ through a wide range of couplings. Notice that for rational frequencies, $\omega \in \mathcal{Q}$ and $\delta \rightarrow \delta_{c}$, for some appropriate value of $\delta_{c}$ which depends on $\omega$, the chain breaks up into a periodic collection of finite segments which naturally yield additional plateaus of rational values. Being the unit cell composed of 21 spins one would naively expect plateaux to appear for values of $M=(2 n+1) / 21, n=0, \ldots, 10$, but as can be seen from Fig. 1 this is not the case due to the nontrivial structure of the unit cell and only some of these values are indeed observed. Interestingly, Eq. (6) yet remains robust all the way through the decoupling point and the corresponding plateau corresponds to the most prominent.

These observations were also corroborated on $X X Z$ chains. However, owing to the large spaces involved in their diagonalization now we content ourselves with moderate lengths $L$ (up to 24 spins). After resorting to the Lanczos method $^{23}$ in each of the magnetization subspaces with $S^{z}$ $\in\{0,1, \ldots, L / 2\}$, we built up the magnetization contours

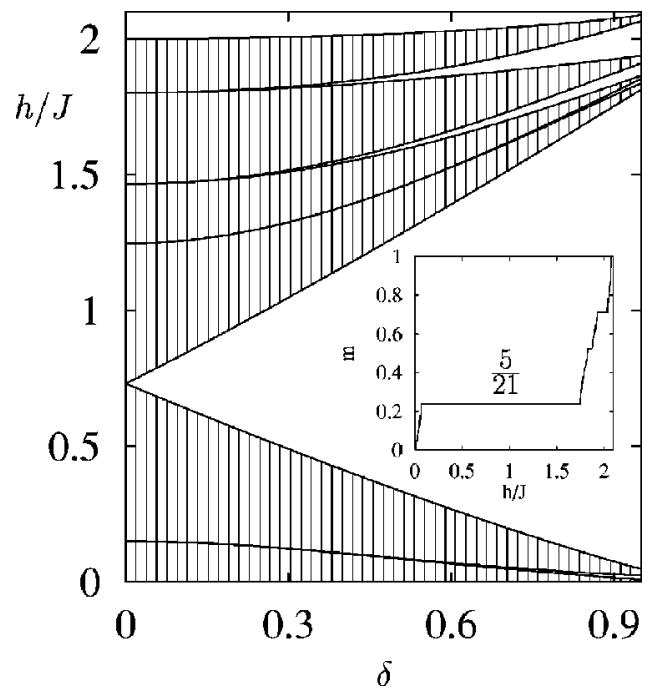

FIG. 1. Critical fields (in bold lines) of the $X X$ chain with $\omega$ $=13 / 21$ and $10^{5}$ spins. At $\delta=0.9$ these fields conform the standard magnetization curve displayed in the inset. Vertical lines denote regions of massless spin excitations where the magnetization increases continuously with $h$. Empty zones in ascending order represent plateaus appearing at $\langle M\rangle=1 / 21,5 / 21$ [central region expected by Eq. (6)], 9/21, 11/21, and 15/21 before saturation (top zone).

shown in Fig. 2 using $\omega=5 / 8$ for $L=8,16,24$. As expected, the massless $X X Z$ excitations around $\langle M\rangle=1 / 4$ render size effects rather noticeable within small coupling regions. Nevertheless, already for $\delta>0.2$ they clearly become less pronounced, thus lending our results further support to the bosonization picture.

A word of caution should be added here, namely, the importance of periodic boundary conditions (PBC's) in testing

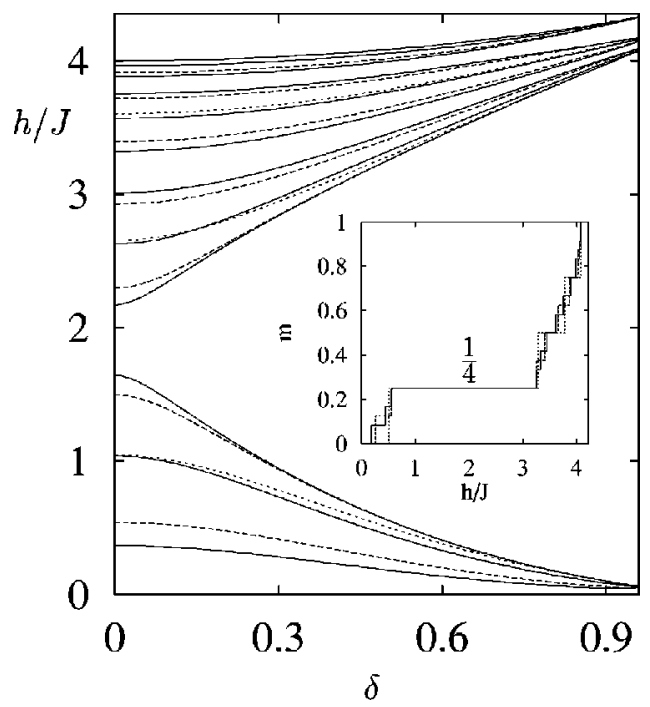

FIG. 2. Magnetization contours of the isotropic $(\Delta=1) X X Z$ chain for $\omega=5 / 8$. Solid, dashed, and dotted lines stand, respectively, for the critical fields of $L=24,16$, and 8 . The middle empty region corresponds to the $\langle M\rangle=1 / 4$ plateau expected by Eq. (6). The inset shows one of the typical magnetization curves upon setting $\delta=0.5$. 


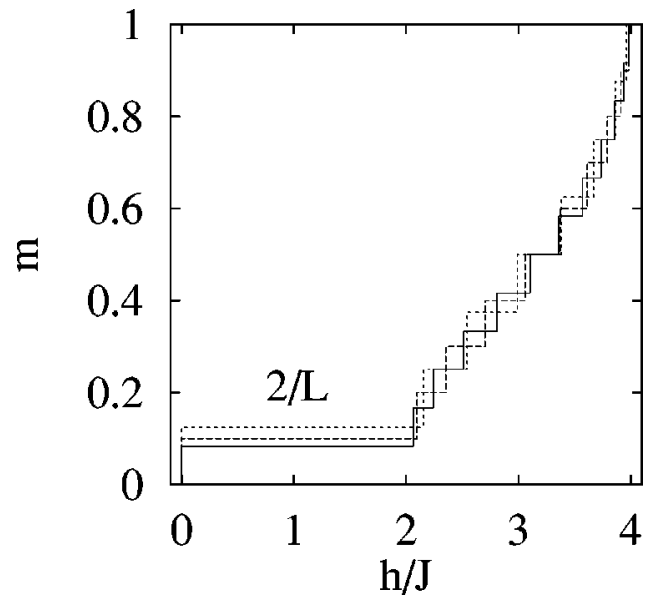

FIG. 3. Magnetization curves of a dimerized Heisenberg chain ( $\omega=1 / 2$ with $\delta=0.4$ ), using open boundary conditions displaying a plateau at $\langle M\rangle=2 / L$. Solid, dashed, and dotted lines denote, respectively, the results of $L=24,20$, and 16 .

the analytic approach via exact diagonalization of small systems. Already at the level of a simple dimerized chain $(\omega$ $=1 / 2$ ), PBC's become crucial. In fact, the numerical analysis of this latter situation using open boundary conditions shows that the well-known $\langle M\rangle=0$ plateau expected in the large length $L$ limit actually emerges at $\langle M\rangle=2 / L$. Figure 3 illustrates this observation for $L=24,20$, and 16 and should emphasize the essential role of PBC's in all our subsequent numerical checks.

By construction, the bosonization approach can be straightforwardly extended to the case in which more than one frequency is present in $\epsilon_{n}$. It turns out that whenever condition (6) is satisfied for each frequency, a magnetization plateau shows up. This extension is simple since each operator (corresponding to each frequency) is commensurate separately, and hence the different perturbations can be treated separately. Of course the situation changes in the case of a dense multifrequency spectrum (such as in the Fibonacci potential that we discuss below) and a more complete analysis has to be carried out. ${ }^{11}$

In order to test the reliability of these predictions, we have analyzed numerically both $X X$ and $X X Z$ chains using double frequency couplings. In Fig. 4 we display the magnetization curves obtained for $\omega_{1}=5 / 8, \omega_{2}=7 / 8$ with amplitudes $\delta_{1}$ $=0.2$ and $\delta_{2}=0.3$, respectively. As we mentioned before, the rather robust plateaus emerging at $\langle M\rangle=1 / 4$ and 3/4 not only confirm the correctness of our extended bosonization prediction, but also pave the way to an alternative description of the massive spin excitations observed in $\mathrm{NH}_{4} \mathrm{CuCl}_{3}$; a quasione-dimensional $S=\frac{1}{2}$ compound which is attracting both theoretical and experimental attention and whose magnetization behavior yet remains unexplained. ${ }^{19,24,25}$ Of course, a more realistic description of this material must start from the microscopic structure observed by $\mathrm{x}$-ray spectroscopy, which points to a two-leg zigzag ladder. Still, it is quite encouraging to obtain a magnetization curve qualitatively similar with the simple two-frequency model we considered here.

In studying irrational frequencies or other quasiperiodic modulations, it is natural to check our analysis with the pro-

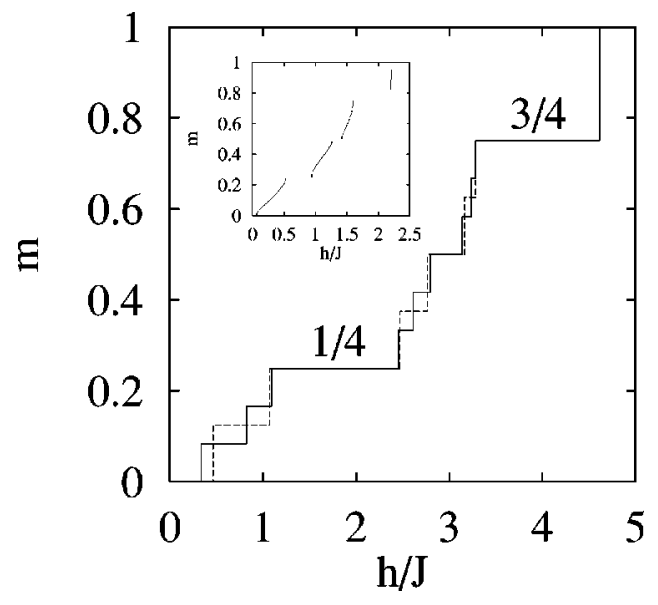

FIG. 4. Double frequency magnetization curve of the isotropic $(\Delta=1) X X Z$ chain for $\omega_{1}=5 / 8, \omega_{2}=7 / 8$ with amplitudes $\delta_{1}=0.2$ and $\delta_{2}=0.3$. Solid and dashed lines denote, respectively, the magnetizations of $L=24$ and 16 . The inset shows the magnetization curve of the corresponding $X X$ chain with $10^{5}$ spins.

totype Fibonacci sequence, a coupling array $J_{A}=J(1+\delta)$, $J_{B}=J(1-\delta)$ generated by iterating the substitution rules $B$ $\rightarrow A$ and $A \rightarrow A B .^{3-5,11}$ Here we discuss the general $X X Z$ situation (a related model has been studied in Ref. 26), and compare our results with the already well-known magnetization curve of the $X X$ case. ${ }^{4,5}$ Before continuing with the bosonization approach, we pause to discuss the strong coupling regimes of this system $(\delta \rightarrow \pm 1)$ in the context of a simple decimation procedure (see, e.g., Ref. 27). To evaluate the magnetization of the widest plateaus, there are two different cases to consider, according to $\delta \simeq-1$, i.e., $J_{B} \gg J_{A}$, and the opposite situation for $\delta \simeq 1$.

Starting from saturation, in the first case the magnetic field is lowered until it reaches the value $h_{c} \simeq J_{B}$ at which the type- $B$ bonds experience a transition from the state of maximum polarization to the singlet state. The magnetization at this plateau is then obtained by decimating the $B$ bonds. This simply yields $\langle M\rangle=1-2 N_{B} /\left(N_{B}+N_{A}\right)$, where $N_{A, B}$ denotes the number of bonds of type $A$ and $B$, respectively. For a large iteration number of the rules referred to above ( $L$ $\rightarrow \infty), N_{A} / N_{B}$ approaches the golden mean $\gamma=(1+\sqrt{5}) / 2$ and therefore we find $M_{1}=(\gamma-1) /(\gamma+1) \simeq 0.236068$.

In the second case, $J_{A} \gg J_{B}$, we have to distinguish two different unit cells since type- $A$ bonds can appear either in pairs (forming trimers) or isolated (forming dimers). It can be readily checked that when lowering the magnetic field from saturation the first spins to be decimated correspond to those forming trimers. We then find two plateaus at $M_{2}=1$ $-2 / \gamma^{3} \simeq 0.527864$ (after decimating trimers) and, like the case $\delta \simeq 1$, at $M_{1}$ (after decimating the remaining dimers). Since the decimation procedure applies for generic $X X Z$ chains ${ }^{27}$ we conclude that the emergence of these strong coupling plateaus is a generic feature, at least with an antiferromagnetic anisotropy parameter $0<\Delta<1$.

For intermediate regimes $0<|\delta|<1$ the magnetization curve has a much richer structure which can be easily understood from our bosonization analysis in a multifrequency case. Evidently, the self-similar hierarchy of frequencies re- 


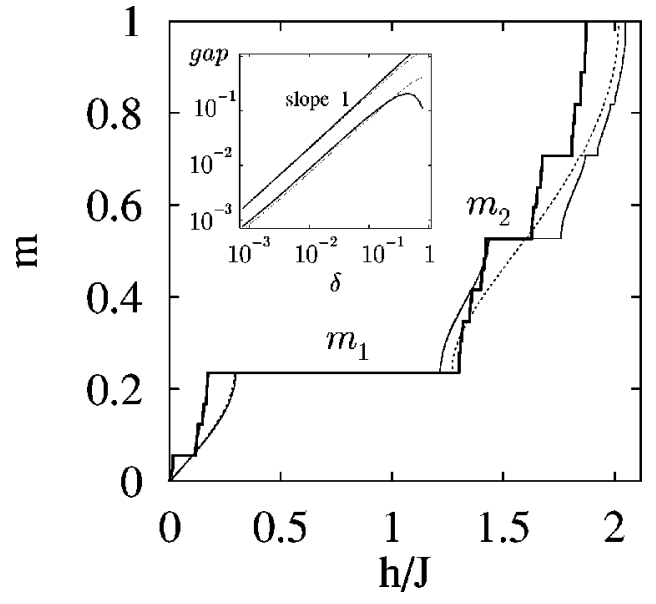

FIG. 5. Fibonacci magnetization for $\delta=0.5$ and $F(25)=75025$ $X X$ spins (bold line). Here, $F(25)$ denotes the 25 th Fibonacci number defined as $F(n+1)=F(n)+F(n-1)$ with $F(1)=F(2)=1$. Dotted and solid lines stand, respectively, for the single and double frequency approximants to the $M_{1}$ and $M_{2}$ plateaus referred to in the text. The slopes compared in the inset show the gap width of $M_{2}$ and $M_{1}$, in ascending order. Bold lines denote the Fibonacci gaps whereas solid lines represent the double approximant widths.

sulting from the Fourier transform of the Fibonacci exchanges (see, e.g., Ref. 11) along with the quantization condition studied so far, enables us to reconstruct the whole spin gap structure of the Fibonacci chain, at least for $|\delta| \ll 1$ and as long as the operator responsible for the plateaus is relevant. Actually, it turns out that one can obtain a fair approximation to the latter showing the most important plateaus by keeping just a few numbers of main spectrum frequencies, even beyond weak-coupling regimes. Interestingly, when the constraint (6) is applied to the dominant Fibonacci frequencies $\omega_{1}=1 / \gamma$ and $\omega_{2}=2(1-1 / \gamma)$ it yields precisely the $M_{1}$ and $M_{2}$ plateaus arising from the strongcoupling decimation. These results are shown in Fig. 5 where both single and double frequency approximations are dis- played. Moreover, using the Fourier spectrum to set $\delta_{2} / \delta_{1}$ $\simeq 0.36237$, the Fibonacci gap widths exhibit a remarkable agreement with the scaling exponents $1 /(2-d)$ referred to above. This can be observed in the inset over more than three decades in $\delta$. In turn, this supports the observation that all gap widths of the $X X$ Fibonacci chain scale simultaneously with $\delta$, as for $\Delta=0$ the compactification radii comprehended in the scaling exponents are independent of the magnetization $[R \equiv 1 /(2 \sqrt{\pi})]$ (see, e.g., Ref. 18).

Finally, we point out that for the Fibonacci chain (which has a dense Fourier spectrum), it was shown by solving the one-loop RG equations at zero magnetic field ${ }^{11}$ (see also Ref. 12) that the critical value of $R$ at which the KosterlitzThouless transition occurs is moved to $R_{c}=1 / \sqrt{4 \pi}$, in contrast to $R_{c}=1 / \sqrt{8 \pi}$ for the single, or more generally nondense frequency case. Hence, for $0<\Delta<1$ and arbitrary magnetization, the operator responsible for spin gap openings at each of the Fibonacci frequencies is relevant. Therefore the magnetization curve of the $X X Z$ case with antiferromagnetic anisotropy is of the same form as that of the $X X$ situation (see Fig. 5), though with different plateau widths.

To summarize, we have studied the interplay between quasiperiodic exchanges and uniform magnetic fields in strongly correlated antiferromagnetic chains using both bosonization and numerical techniques. The former were tested and complemented by the latter in a variety of nonperturbative scenarios. Our calculations suggest the possibility to observe rather stable magnetization plateaus [Eq. (6)] on artificially grown arrays of quantum $\operatorname{dots}^{9}$ according to the (controlled) spatial distribution of their exchange integrals. We trust this work will convey an interesting motivation for further experimental studies in these material technologies.

It is a pleasure to acknowledge useful discussions with A. Honecker and P. Pujol. The research of D.C.C. and M.D.G. was partially supported by CONICET and Fundación Antorchas, Argentina (Grant No. A-13622/1-106).
${ }^{1}$ D. Schechtman, I. Blech, D. Gratias, and J. W. Cahn, Phys. Rev. Lett. 53, 1951 (1984).

${ }^{2}$ C. Godrèche, J. M. Luck, and H. Orland, J. Stat. Phys. 45, 777 (1986)

${ }^{3}$ Y. Achiam, T. C. Lubensky, and E. W. Marshall, Phys. Rev. B 33, 6460 (1986).

${ }^{4}$ J. M. Luck and Th. M. Nieuwenhuizen, Europhys. Lett. 2, 257 (1986).

${ }^{5}$ M. Kohmoto, B. Sutherland, and C. Tang, Phys. Rev. B 35, 1020 (1987)

${ }^{6}$ T. J. Sato, H. Takakura, A.P. Tsai, K. Shibata, K. Ohoyama, and K.H. Andersen, Phys. Rev. B 61, 476 (2000).

${ }^{7}$ D. C. Cabra, M. D. Grynberg, A. Honecker, and P. Pujol, cond-mat/0010376 (unpublished).

${ }^{8}$ R. Merlin, K. Bajema, R. Clarke, F.-Y. Juang, and P. K. Bhattacharya, Phys. Rev. Lett. 55, 1768 (1985).

${ }^{9}$ L. P. Kouwenhoven, F. W. J. Hekking, B. J. van Wees, C. J. P. M.
Harmans, C. E. Timmering, and C. T. Foxon, Phys. Rev. Lett. 65, 361 (1990).

${ }^{10}$ C. G. Bezerra, J. M. de Araujo, C. Chesman, and E. L. Albuquerque, cond-mat/0101099 (unpublished).

${ }^{11}$ J. Vidal, D. Mouhanna, and T. Giamarchi, Phys. Rev. Lett. 83, 3908 (1999).

${ }^{12}$ K. Hida, Phys. Rev. Lett. 86, 1331 (2001); K. Hida, J. Phys. Soc. Jpn. 68, 3177 (1999); 69, 311 (2000).

${ }^{13}$ W. Chen, K. Hida and H. Nakano, J. Phys. Soc. Jpn. 68, 625 (1999).

${ }^{14}$ R. M. Wiessner, A. Fledderjohann, K.-H. Mütter, and M. Karbach, Eur. Phys. J. B 15, 475 (2000).

${ }^{15}$ O. Derzhko, J. Richter, and O. Zaburannyi, J. Magn. Magn. Mater. 222, 207 (2000).

${ }^{16}$ M. Oshikawa, M. Yamanaka, and I. Affleck, Phys. Rev. Lett. 78, 1984 (1997).

${ }^{17}$ K. Totsuka, Phys. Lett. A 228, 103 (1997). 
${ }^{18}$ D. C. Cabra, A. Honecker, and P. Pujol, Phys. Rev. Lett. 79, 5126 (1997); Phys. Rev. B 58, 6241 (1998).

${ }^{19}$ H. Tanaka, K. Takatsu, W. Shiramura, T. Kambe, H. Nojiri, T. Yamada, S. Okubo, H. Ohta, and M. Motokawa, Physica B 246, 230 (1998); W. Shiramura, K. Takatsu, B. Kurniawan, H. Tanaka, H. Uekusa, Y. Ohashi, K. Takizawa, H. Mitamura, and T. Goto, J. Phys. Soc. Jpn. 67, 548 (1998).

${ }^{20}$ A. O. Gogolin, A. A. Nersesyan, and A. M. Tsvelik, Bosonization of Strongly Correlated Systems (Cambridge University Press, Cambridge, England, 1998).

${ }^{21}$ T. Hikihara and A. Furusaki, Phys. Rev. B 58, R583 (1998).

${ }^{22}$ S. Lukyanov and A. Zamolodchikov, Nucl. Phys. B 493, 571
(1997).

${ }^{23}$ Consult, for example, G. H. Golub and C. F. Van Loan, Matrix Computations, 3rd ed. (Johns Hopkins University Press, Baltimore, 1996).

${ }^{24}$ A. K. Kolezhuk, Phys. Rev. B 59, 4181 (1999).

${ }^{25}$ D. C. Cabra and M. D. Grynberg, Phys. Rev. B 62, 337 (2000).

${ }^{26}$ T. Tokihiro, Phys. Rev. B 41, 7334 (1990).

${ }^{27}$ D. C. Cabra, A. De Martino, M. D. Grynberg, S. Peysson, and P. Pujol, Phys. Rev. Lett. 85, 4791 (2000); D. S. Fisher, Phys. Rev. B 50, 3799 (1994); S. K. Ma, C. Dasgupta, and C.-K. Hu, Phys. Rev. Lett. 43, 1434 (1979); C. Dasgupta and S. K. Ma, Phys. Rev. B 22, 1305 (1979). 